\title{
Acute Infection in Total Knee Arthroplasty: Diagnosis and Treatment
}

\author{
Juan Carlos Martínez-Pastor ${ }^{*}$, Francisco Maculé-Beneyto and Santiago Suso-Vergara
}

\author{
Department of Orthopaedics and Trauma Surgery, Bone and Joint Infection Unit, Hospital Clinic, University of \\ Barcelona, C/Villarroel 170, 08036, Barcelona, Spain
}

\begin{abstract}
Infection is one of the most serious complications after total knee arthroplasty (TKA). The current incidence of prosthetic knee infection is $1-3 \%$, depending on the series For treatment and control to be more cost effective, multidisciplinary groups made up of professionals from different specialities who can work together to eradicate these kinds of infections need to be assembled. About the microbiology, Staphylococcus aureus and coagulase-negative staphylococcus were among the most frequent microorganisms involved (74\%).

Anamnesis and clinical examination are of primary importance in order to determine whether the problem may point to a possible acute septic complication. The first diagnosis may then be supported by increased CRP and ESR levels. The surgical treatment for a chronic prosthetic knee infection has been perfectly defined and standardized, and consists in a two-stage implant revision process. In contrast, the treatment for acute prosthetic knee infection is currently under debate. Considering the different surgical techniques that already exist, surgical debridement with conservation of the prosthesis and polythene revision appears to be an attractive option for both surgeon and patient, as it is less aggressive than the twostage revision process and has lower initial costs.

The different results obtained from this technique, along with prognosis factors and conclusions to keep in mind when it is indicated for an acute prosthetic infection, whether post-operative or haematogenous, will be analysed by the authors.
\end{abstract}

Keywords: Acute haematogenous infections, Acute post-operative infections, Biofilm, Implant retention, Open debridement, Total knee arthroplasty.

\section{INTRODUCTION}

Infection is one of the most serious complications after total knee arthroplasty (TKA). This issue is not only a challenge to the orthopaedic surgeon but also to the patient and their families in terms of the physical and psychological impacts that are normally associated with this problem. It not only affects their quality of life but also their ability to return to their daily routine and leads to decreased functionality and quality of life results.

Another important aspect of prosthetic infection is its serious economic impact. The cost of curing this type of infection is approximately $\$ 70,000$ dollars [1] per patient depending on the number of surgeries, complications and antibiotic treatments the patient requires. In general, when prosthesis becomes infected, three to four times as many resources are required in comparison to a primary knee arthroplasty, and twice as many when compared to a prosthesis revision of aseptic etiology.

For treatment and control to be more cost effective, multidisciplinary groups made up of professionals from different specialities who can work together to eradicate these kinds of infections need to be assembled. The improved cost-effectiveness must begin with prophylaxis control, early diagnosis and establishment of clear protocols

*Address correspondence to this author at the Department of Orthopaedics and Trauma Surgery, Bone and Joint Infection Unit, Hospital Clinic, University of Barcelona, C/Villarroel 170, 08036, Barcelona, Spain; Tel: +34932275400, Ext. 5533; E-mail: jcmartin@clinic.ub.es for the various situations or for presenting symptoms under which prosthetic knee infections might appear.

According to published studies, the current incidence of prosthetic knee infection is $1-3 \%$, depending on the series [2], and antibiotic prophylaxis is the most effective method in reducing prosthetic joint infection rates.

\section{RISK FACTORS ASSOCIATED WITH PROSTHETIC KNEE INFECTIONS}

Various types of factors may play a role in the appearance of a prosthetic infection. On the one hand there are factors that follow solely from the anatomic location of the knee joint which, due to its very superficial nature and scant muscle coverage, can result in more cutaneous complications than arthroplasties in other sites.

On the other hand, there are factors that depend on the host, such as immunosuppression in the case of a transplant patient or one who suffers from rheumatoid arthritis. A greater risk has also been observed among diabetes mellitus patients and obese patients [3,4] two conditions which are often related.

Pre-existing septic arthritis also increased the risk of infection after prosthesis was implanted in the same joint. Prior surgical interventions to the primary prosthetic implant and prosthetic revision surgeries further increase the risk of developing a prosthetic infection.

Total control of the environment within the operating room is very important during a prosthetic knee replacement. The number of people in the operating room should be 
reduced to the minimum number required, and continual entering and leaving of the operating room should be avoided. Using a device such as ultraviolet light or laminar flow can contribute to a reduction in the number of prosthetic infections.

With regards to treatment of the surgical field, recent studies [5] have suggested that pre-operative skin sterilisation with chlorhexidine may provide better results towards avoiding infection than the traditional use of povidone-iodine.

\section{MICROBIOLOGY}

Table 1 lists the microorganisms most frequently related to prosthetic infection, according to the results obtained from primary prosthetic knee infections (cemented without local antibiotics) performed at the Hospital Clinic of Barcelona in Spain from 2007 to 2009. Staphylococcus aureus and coagulase-negative staphylococcus (74\%) were among the most frequent microorganisms involved.

Table 1. Microbiological Results in Prosthetic Knee Infections Diagnosed at Hospital Clinic of Barcelona (Spain) from 2007 to 2009

\begin{tabular}{|c|c|}
\hline Microorganism (\%) & Primary TKA (Without Local Antibiotic) \\
\hline \hline S. aureus & $19(55.8 \%)$ \\
\hline ECN & $6(17.6 \%)$ \\
\hline E. faecalis & $3(8.8 \%)$ \\
\hline Corynebacterium & 0 \\
\hline E. coli & $3(8.8 \%)$ \\
\hline E. cloacae & $1(2.9 \%)$ \\
\hline K. pneumoniae & $1(2.9 \%)$ \\
\hline P. aeruginosa & $1(2.9 \%)$ \\
\hline
\end{tabular}

Depending on the infection's presenting symptoms, one form or another of gram-positive bacteria will predominate. For example, in acute prosthetic infections there is a greater prevalence of $S$. aureus bacteria followed by coagulasenegative staphylococcus. The order of prevalence changes when considering chronic prosthetic infections, in which case coagulase-negative staphylococcus predominates over S. aureus. Gram-negative microorganisms are found in about $17.6 \%$ of prosthetic infections. Twenty five of the infections tend to be a combination of various types of microorganisms, commonly referred to as polymicrobial infections.

Fungal infections are less frequent, most of which are caused by Candida saprophyticus (spp). Controlling these types of infections can become even more difficult if they appear in combination with other types of bacterial infections [6]. As will be seen below, the type of microorganism that causes the infection can be a key factor in the development and prognosis of acute prosthetic knee infections.

\section{PATHOPHYSIOLOGY}

The main characteristic of prosthetic infections is the formation of a biofilm on the surface of the implants. The relapse rate that is observed in orthopaedic implant infections, even after very prolonged treatment is due to the formation of this biofilm.

The bacteria assemble themselves within this biofilm in different strata as each has distinct metabolic activities. At the same time, these bacteria secrete an extracellular polymeric substance (EPS) [7] that helps the bacteria that are located in the deepest strata of the biofilm to survive in reduced metabolic conditions with little available nutrients. These are the bacteria that have the highest resistance to antibiotics, as opposed to those bacteria that are located on the biofilm's surface. Some authors believe that the difference between an acute and a delayed infection could be related to the maturity and extension of the biofilm relative to the time over which it has developed.

\section{PROSTHETIC INFECTION CLASSIFICATION}

Prosthetic infections have traditionally been divided into 4 types [8]:

1. Acute post-operative infections ( $<=4$ weeks postop).

2. Delayed chronic infections.

3. Acute haematogenous infections.

4. Intra-articular positive cultures (no clinical suspicion of infection but two or more positive cultures of the same germ are isolated during surgery).

In clinical practice we classify them into two larger groups, as either acute or chronic infections. As can be seen in the prior classification, acute infections can be divided into two very distinct groups:

1.- Acute post-operative infections are those infections that occur during surgery and usually appear from the moment the operation was performed up to three months later, depending on the microorganism responsible for the infection. They are usually diagnosed within the first month.

2.- Acute haematogenous infections or Delayed acute infections are those infections that appear suddenly long after the placement of the implant. The patient does not normally mention any problems with the prosthesis and has a high level of functionality until a sudden pain appears along with joint effusion which may be preceded by chills that are a result of bacteraemia. The focal point of the infection is typically located at a certain distance from the prosthesis, such as in the urinary system, or else may originate from a cutaneous wound or a vascular catheter. This would be a typical example of the clinical symptoms from an acute haematogenous infection.

In other cases the appearance of infection is acute and there is a window period during which the patient shows no symptoms of infection, but it is impossible to isolate the focal point from which the infection is originating from. As a result, a direct relationship between cause and effect cannot be established. These types of infections are the ones which some authors refer to as delayed acute infections [9].

\section{ACUTE PROSTHETIC KNEE INFECTION DIAGNOSIS}

Diagnosing an acute prosthetic knee infection is mainly based on the symptoms and clinical signs experienced by the 
patient such as pain, erythema, effusion, signs of inflammation and persistent drainage from the surgical wound. Some studies have found that for arthroplasties in general, including hip arthroplasties, persistent drainage from the surgical wound is associated with $15-50 \%$ of deep tissue infections. If knee arthroplasties are considered more specifically, the presence of drainage from the surgical wound over a long period of time, even when the fluid is serous, should be treated surgically with minimal delay since it is a high indicator of prosthetic infection. This might be explained by the knee's anatomical location, with little subcutaneous cellular tissue or muscle coverage, as a result of which the drainage would most likely originate from inside the joint.

\section{Serological Tests}

a. The leukocytic count in a blood test does not typically lend any information for diagnosing an acute postoperative infection since it is usually normal. This indicator is more helpful when diagnosing acute haematogenous infections, particularly in cases where symptoms of bacteraemia have appeared and blood cultures have been positive. In these cases the leukocytic count in peripheral blood is elevated and neutrophils might even appear.

b. Erythrocyte sedimentation rate (ESR) in an acute post-operative infection is very unspecific since it is only a marker of general inflammation that tends to return to normal within 3 months to a year after the prosthetic surgery. In chronic infections, the ESR also returns to normal within 3 months to a year after the surgery. After the third month the $22.5 \mathrm{~mm} / \mathrm{h}$ threshold has $93 \%$ sensitivity and $83 \%$ specificity [10].

c. C-reactive protein (CRP) is an acute-phase reactant that is also non-specific for prosthetic infections since it can rise in response to other kinds of infections, traumatisms or any other kind of inflammatory processes. It is important to keep in mind that CRP usually takes between 2 and 3 weeks to return to base levels, which is why this parameter is difficult to interpret in acute post-operative infections. CRP levels below $6 \mathrm{mg} / \mathrm{dl}$ on the $5^{\text {th }}$ day after surgery do seem relevant, as they are considered a sign of absence of complications due to the operation [11].

d. Recent investigations are considering other inflammation markers such as IL-6 [12]. IL-6 is also an acute-phase reactant that peaks 6 to 12 hours after prosthetic surgery but has the advantage, in comparison to the previous two markers, of returning to normal levels between 48 and 72 hours after the surgery. This means that the use of IL- 6 could be of greater use in the diagnosis of acute infections in comparison to the other two.

\section{Radiology}

Radiological images of acute post-operative infections do not usually show loosening or lytic lesions at the metaphyseal level, as these are typically indicative of a chronic septic process. However, they are useful to assure that components are in the correct position and that the limb is well-aligned, which is helpful towards bringing into focus the surgical treatment that will later be required. In acute haematogenous infections radiological images are more useful, especially in cases where the prosthesis has been in place for a long period of time. In these cases, radiolucent lines can appear that bring into question the stability of the implant, and can be helpful towards determining the surgical treatment to be carried out (Fig. 1).

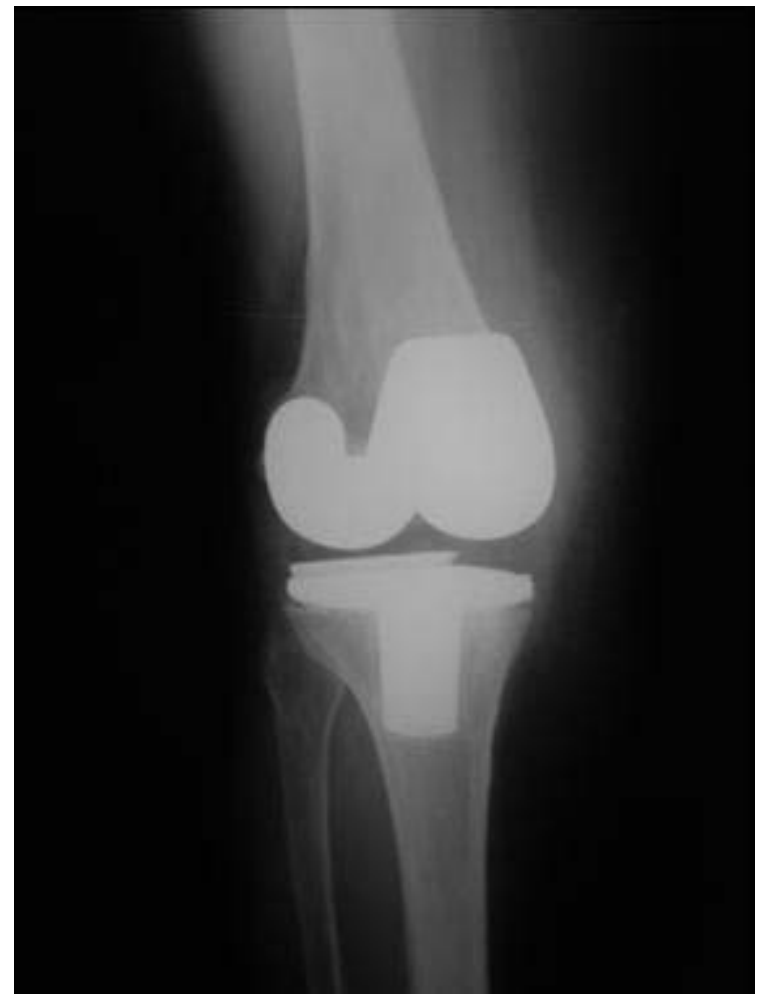

Fig. (1). 87-year-old patient with an acute haematogenous infection caused by Listeria monocytogenes. The radiolucent lines can be observed around the tibial component.

\section{Leukocyte-Labelled Bone Scintigraphy}

According to the recommendations made by the American Academy of Orthopaedic Surgeons about prosthetic infections (AAOS Clinical Practice Guideline Summary. Diagnosis of Periprosthetic Joint Infections of the Hip and Knee. 2011), bone scintigraphy is of weak value and would only be recommended in very specific cases. Above all, in cases where chronic prosthetic infection with certain specific characteristics is suspected (Recommendation 9). In the diagnosis of an acute prosthetic infection, leukocytelabelled bone scintigraphy does not yield specific results and is therefore not recommended.

\section{Joint Aspiration}

If an acute infection of a knee prosthesis is suspected, joint aspiration is a quick and recommended test.

It is important to maintain an antibiotic-free window period of approximately 15 days prior to the test (AAOS Clinical Practice Guideline Summary. Diagnosis of Periprosthetic Joint Infections of the Hip and Knee. 2011. Recommendation 8).

The material extracted should be sent for biochemical and microbiological analysis. For the latter, it is 
recommended that the material be inoculated into hemoculture flasks, since some authors have found that this medium yields better results towards microorganism identification [13].

In terms of cellular counts, the parameters established by some authors [14], with a threshold for leukocytic count of $1100 \mathrm{WBC} / \mathrm{ml}$, have yielded $91 \%$ sensitivity and $88 \%$ specificity. A differential count (percentage of neutrophils) > $64 \%$ is associated with $95 \%$ sensitivity and $94 \%$ specificity. It must be mentioned that these indicators are especially useful for chronic prosthetic infections. The biochemical study of the fluid obtained from joint aspiration of the acute prosthetic infection usually shows higher levels of leukocytes and the differential count very often remains above $90 \%$.

Recent studies carried out by Parvizi et al. [14], are considering the value of C-reactive protein in synovial fluid as a prosthetic knee infection predictor with good results. In this particular study, 63 patients were divided into two groups: a septic group (20) and an aseptic group (40). Significant differences were found between the two. In the septic group, the average level was $40 \mathrm{mg} / \mathrm{L}$ as opposed to 2 $\mathrm{mg} / \mathrm{L}$ in the aseptic group $(\mathrm{P}<0.001)$. The sensitivity was $85 \%$ with $95 \%$ specificity at a threshold of $9.5 \mathrm{mg} / \mathrm{L}$.

To conclude this section, it must be mentioned that anamnesis and clinical examination are of primary importance in order to determine whether the problem may point to a possible acute septic complication. The first diagnosis may then be supported by increased CRP and ESR levels. It is important to hold off on empirical antibiotic treatment when an infection is suspected, especially if joint fluid has not been previously obtained and sent for microbiological and biochemical analysis. In the case of our study, unless a patient's blood work showed sepsis or had a fever, no antibiotics were administered until the surgery was performed, which was done in a timely manner.

In most cases, confirmation of an acute prosthetic infection is obtained largely from the intraoperative cultures and the macroscopic evidence of the infection's spread inside the joint. Infections in the surgical wound of prosthetic knee surgeries should be considered deep tissue infections, which is to say that the implant itself has been affected in the large majority of cases. Only in specific cases can an infection be considered superficial, namely when it is related to the suture staples. In this case the problem is usually resolved once the staples are removed.

In acute haematogenous infections or delayed infections, joint aspiration is more frequently purulent than in acute post-operative infections. As we shall see, the presence of pus has a negative predictive value in terms of conserving the implant (Fig. 2).

\section{CRITERIA FOR DEFINING PROSTHETIC INFECTION}

In association with clinical symptoms, different studies have proposed different diagnostic criteria [15], and a new definition for prosthetic infection has even been proposed. This new definition is based on the following premises:

1. Presence of fistulae, or
2. 2 or more positive cultures for the same microorganism, or

3. Presence of 4 of the following:

\section{Elevated CRP or ESR}

Elevated cell counts in the synovial fluid

Elevated percentage of neutrophils in the synovial fluid

1 positive intra-articular culture

$>5$ neutrophils / 5 fields in the pathology study

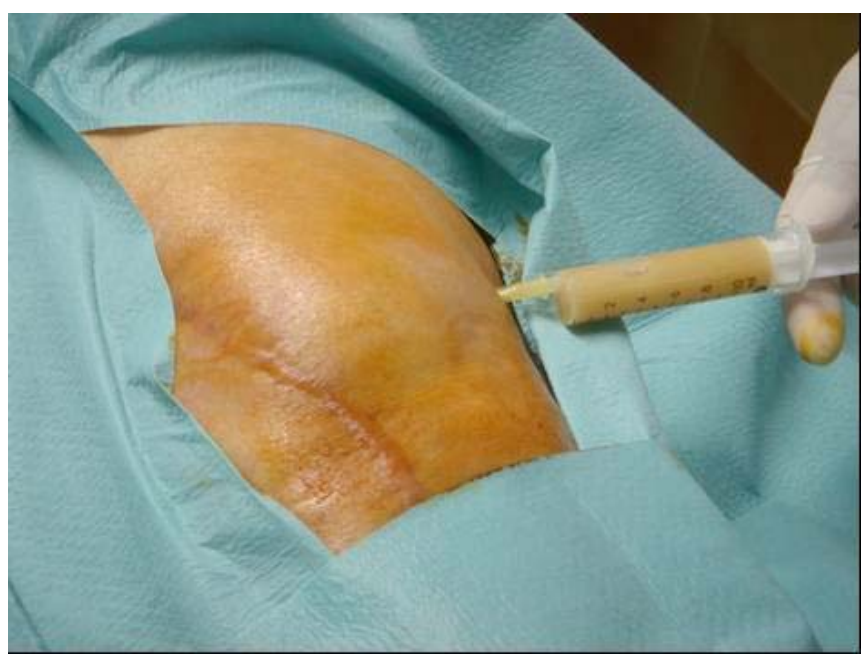

Fig. (2). An Arthrocenthesis with pus.

\section{TREATMENT}

The surgical treatment for a chronic prosthetic knee infection has been perfectly defined and standardized, and consists in a two-stage implant revision process [16]. In the first surgical stage, the prosthesis is extracted and an attempt is made to conserve the largest amount of bone stock as possible. Next, an exhaustive surgical cleaning is carried out, removing all sphacelous tissue or tissue that is suspected of being infected. Finally, an antibiotic-loaded cement spacer is implanted (Figs. 3, 4).

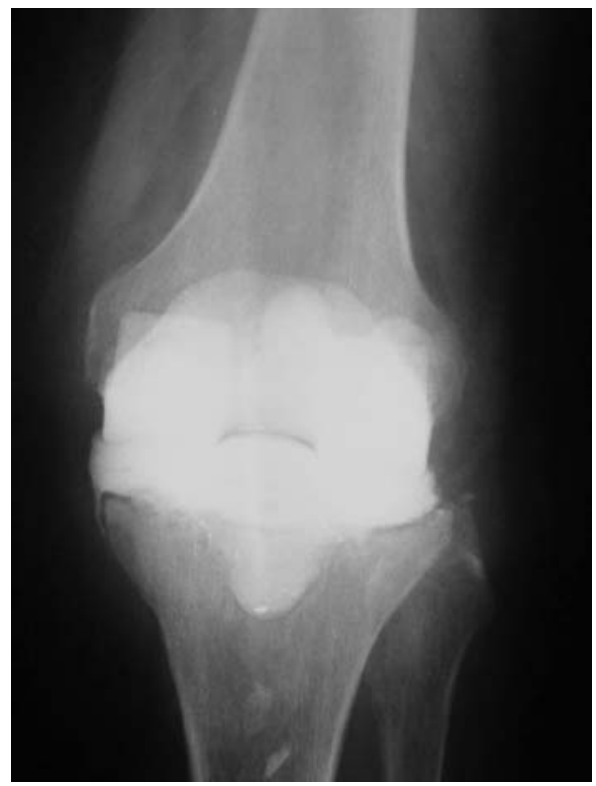

Fig. (3). Anteroposterior X-ray of the joint spacer. 


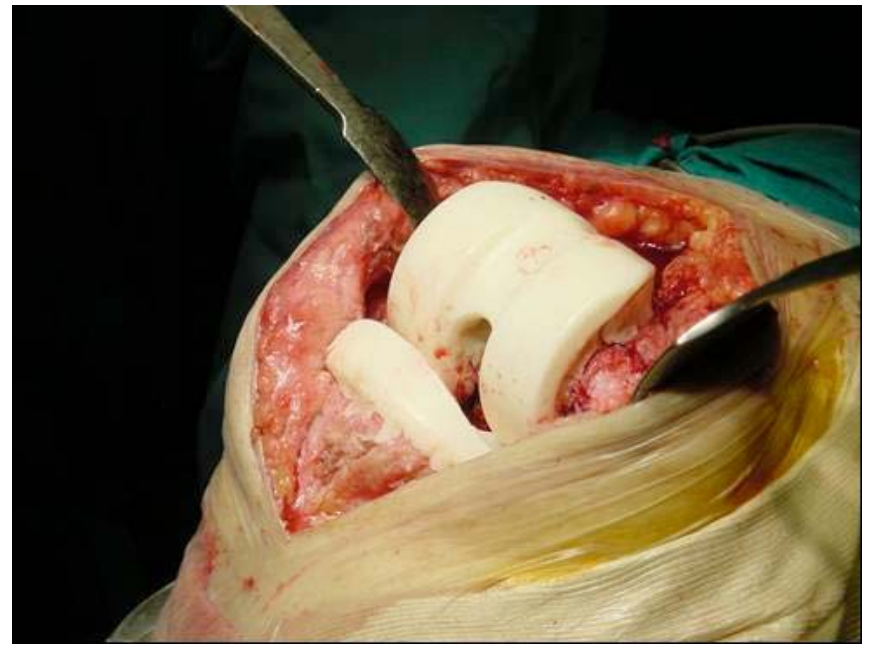

Fig. (4). Anteroposterior X-ray of the joint spacer. Surgical detail of the joint spacer.

After a period of antibiotic treatment and once analytic parameters have normalised (CRP and ESR), a new surgery is performed (second stage) which consists in extracting the spacer and implanting the revision prosthesis.

In contrast, the treatment for acute prosthetic knee infection is currently under debate. Considering the different surgical techniques that already exist, surgical debridement with conservation of the prosthesis and polythene revision appears to be an attractive option for both surgeon and patient, as it is less aggressive than the two-stage revision process and has lower initial costs. The debate stems from the view of some authors that this surgical technique, which retains the implants, does not provide satisfactory results in terms of controlling the infection, and instead advocate for prosthetic revision techniques. The different results obtained from this technique, along with prognosis factors and conclusions to keep in mind when it is indicated for an acute prosthetic infection, whether post-operative or haematogenous, will be analysed by the authors further below.

\section{Surgical Debridement Technique Used on an Acute Prosthetic Knee Infection. Hospital Clinic Universitario de Barcelona Protocol}

The surgical cleaning performed for retaining prosthetic knee implants consists of two distinct parts:

\section{First Part}

The patient is placed in a supine decubitus position and ischemia is performed. The incision into the skin is made iteratively over the pre-existing line, attempting to surround dehiscent areas and devitalised margins. Prior to the arthrotomy the joint is punctured and joint fluid is obtained for microbiological study in blood culture bottles (Bactec 9240 System). (Fig. 5)

Next, arthrotomy and synovectomy of the quadricipital pouch and lateral recesses are carried out, along with removal of all the sphacelous intra-articular tissue that shows signs of infection (Fig. 6).

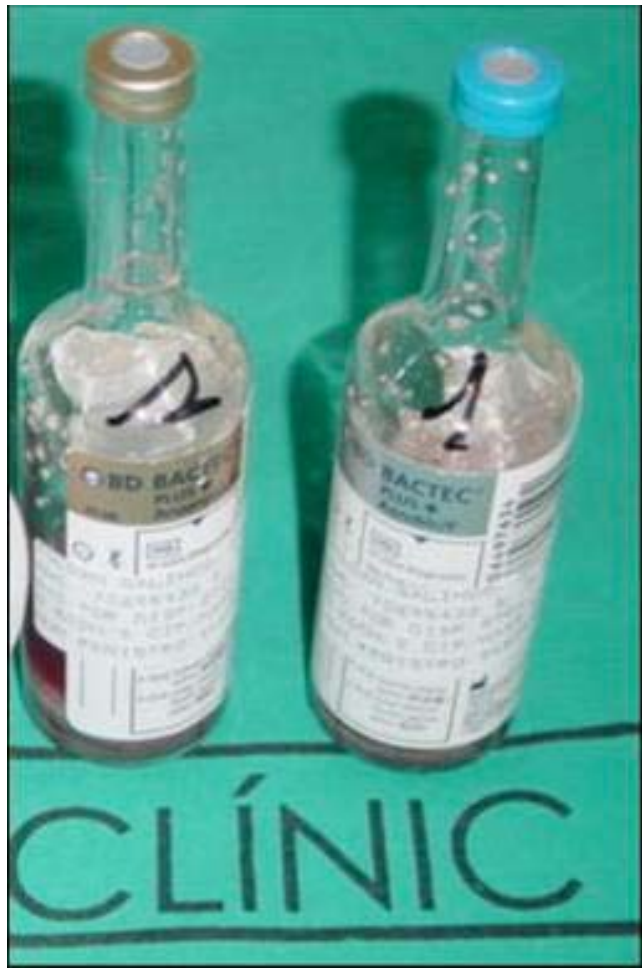

Fig. (5). Blood culture bottle with medium.

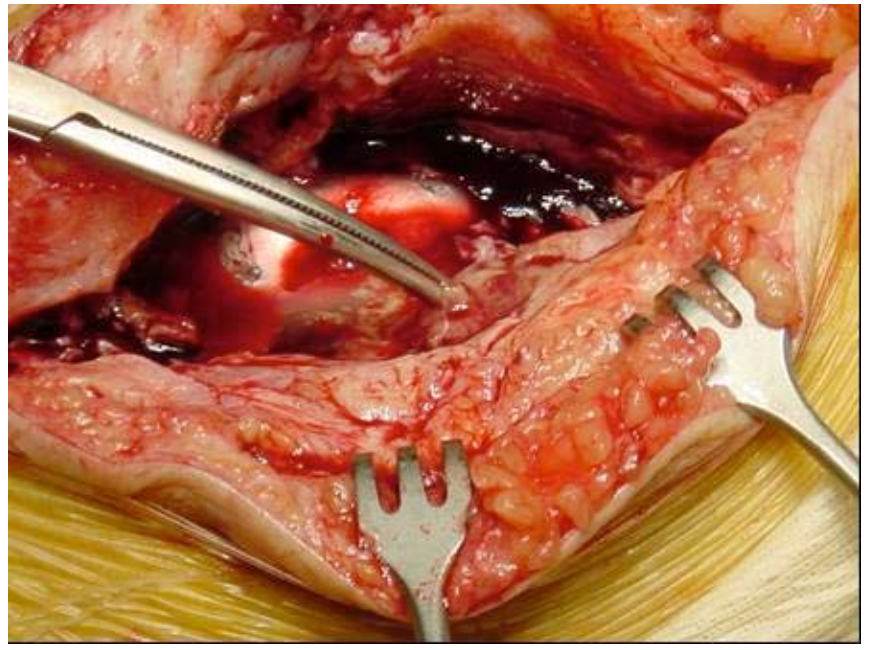

Fig. (6). Synovectomy in a case of Acute Periprosthetic Infection of the Knee.

Once the polythene, if modular, has been removed, the entire posterior capsule is cleaned as well. The rest of the samples for the microbiological tests are taken ( 2 smears and 2 solid material samples, making for a total of 6 samples including the blood culture bottles) as well as samples for pathology. As soon as the samples have been taken antibiotic prophylaxis is administered (according to the Septic Unit of the Hospital Clinic Universitario de Barcelona protocol this consists of teicoplanin and ceftazidime). At this point it is important to mention that even though, in the case of acute prosthetic infections, we administer antibiotic prophylaxis after the samples are taken, some authors have shown that antibiotic prophylaxis at the time of induction does not alter 
the results of the microbiological cultures obtained during the surgery [17]. Following debridement, lavage irrigation is performed with 9 litres of saline solution (Fig. 7).

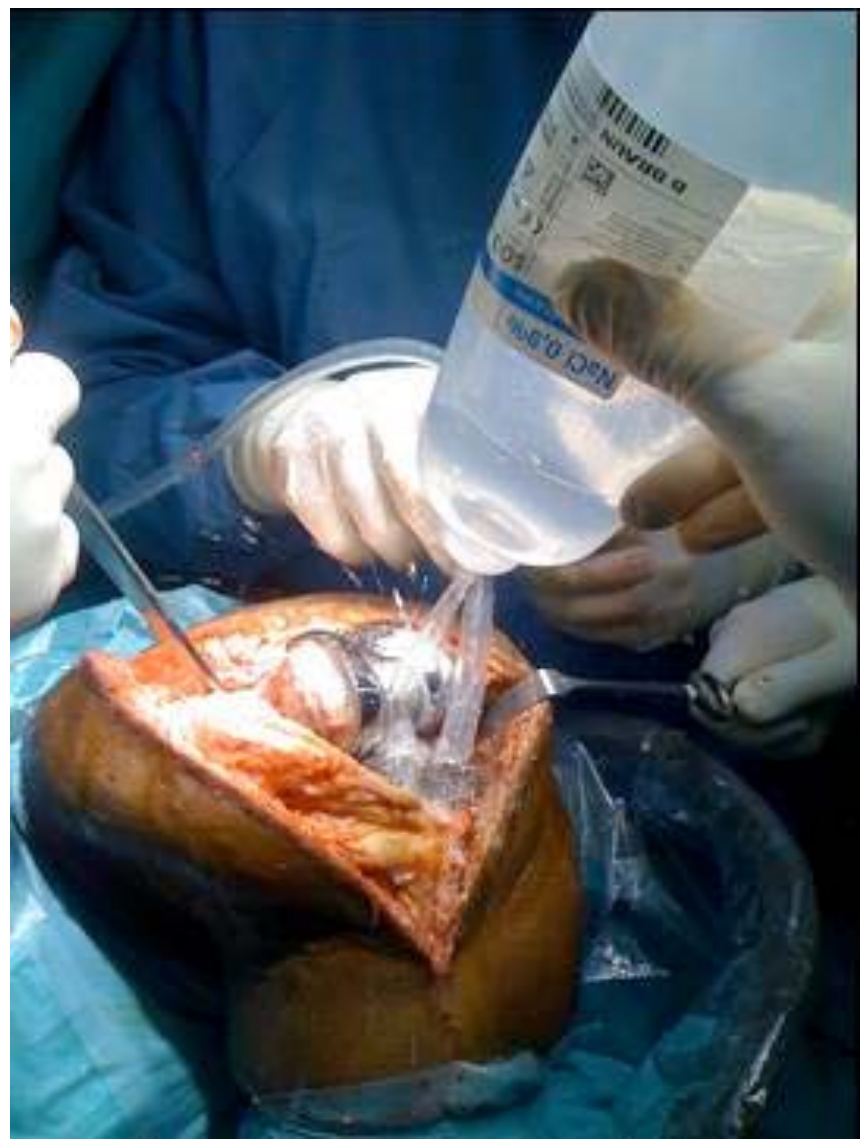

Fig. (7). Irrigation with 9 litres of saline solution, performed just after synovectomy.

Pulsatile lavage irrigation system for prosthetic infections is controversial. In vitro studies confirm that this method of irrigation causes the microorganisms to penetrate into the tissues and is therefore questionable in terms of eradicating the infection. In our unit we have carried out a comparative study on acute implant infections (including osteosynthesis, knee and hip prosthesis materials) and no significant differences were found between physiological saline solution irrigation with a spray gun and saline solution applied directly from sterile bottles [18].

\section{Second Part}

Once the irrigation is complete the second part begins:

A new instrument table and set of surgical tools are used, the surgeons' gloves and coats are changed and a new preparation of the surgical field is made so that the new polythene can be placed in the most sterile conditions possible. Upon placing the polythene, the ischemia is reverted and haemostasis of the surgical field is carried out followed by closure of the surgical wound. After the surgery the patient begins empirical intravenous treatment which, according to our protocols, consists in vancomycin and ceftazidime until definitive microbiological results are obtained from the corresponding antibiogram.

\section{DISCUSSION}

As has already been mentioned, the surgical treatment of acute prosthetic knee infection by surgical debridement and implant retention is currently under debate. Despite the fact that this was once considered the treatment of choice for these types of infections, recent studies are questioning its success towards controlling infection [19]. After surgery, the antibiotic treatment has not been standardised, either with respect to length of treatment or route of administration, and oral treatment has also been questioned.

If we review the existing literature we can see that success rates with this procedure are variable. Segawa $e t$ al. [20] was able to manage an infection control rate of $78 \%$ for infections treated within the first month.

Mont et al. [21] achieved a 100\% cure rate for surgically treated infections with debridement within the first 4 weeks after the prosthesis was implanted. In contrast, other authors such as Silva et al. [22], only had a $32 \%$ success rate in patients treated with this method. Koyonos et al. [9], after reviewing a total of 153 patients treated with surgical debridement between 1996 and 2007 found a 40\% infection control rate.

In the previously mentioned studies carried out by Segawa et al. [20] and Mont et al. [21], the duration of symptoms or the time elapsed after implantation of the prosthesis have been considered prognosis factors for control of the infection. In support of this, Brandt et al. [22] show that prostheses that were debrided after two days of the appearance of clinical symptoms resulted in a high rate of failure, with time elapsed being a prognostic factor towards control of the infection.

However, other studies such as Azzam et al. [23] and Koyonos et al. [9] report no significant relation between success rates and duration of clinical symptoms or the time from index surgery.

In the series on acute post-operative infections treated with surgical debridement and implant retention carried out by the Department of Traumatology and Orthopaedics at the Hospital Clinic [24] since the year 2000, no significant differences were found with regards to the time from index surgery and infection control.

Few studies exist that compare success rates between the acute post-operative and acute haematogenous infections. Acute haematogenous infections, according to authors such as Vilchez et al. [24], show more obvious clinical and laboratory signs of prosthetic infection, and moreover result in lower success rates $(50 \%)$ than acute post-operative infections, especially so when the infection is caused by $S$. aureus.

Among the factors of poor prognosis for acute prosthetic knee infections treated with surgical debridement and implant retention, the following have been described:

1. Old age [25].

2. The American Society of Anesthesiology Scale (ASA) [23], with ASA III and IV patients presenting higher rates of failure for infection control. 
3. The presence of intra-articular pus [23], as this indicates established infection with devitalised tissues and a wider extension around the periprosthetic tissues.

4. The type of microorganism $[9,23,25]$ also appears to influence infection control.

Among the acute infections produced by gram-positive cocci that were treated with surgical debridement and implant retention, various studies show higher success rates with infections resulting from coagulase-negative staphylococci and streptococci [25].

In contrast, some studies have reported Staphylococcus aureus to be an independent factor of poor prognosis with regards to acute infection control with surgical debridement $[9,23,25]$.

According to these authors, in those cases where the infection is caused by Staphylococcus aureus, and particularly so when it is a methicillin-resistant variety, the total removal of the implant should be considered [19,25].

In the review performed at our department of acute infections caused by Staphylococcus aureus from 2000 to 2007 and that were treated with surgical debridement and implant retention, a $75 \%$ infection control rate was observed after two years of follow-up [24]. After the debridement the patients began empirical wide-spectrum antibiotic treatment with vancomycin and ceftazidime. If Staphylococcus aureus had already been isolated in the joint fluid prior to the surgical intervention, vancomycin was substituted for intravenous cloxacillin. After 10 days of intravenous antibiotic treatment, oral antibiotics were administered. In most cases the antibiotics prescribed were levofloxacin $(500 \mathrm{mg} / 12 \mathrm{~h})$ and rifampicin $(600 \mathrm{mg} / 24 \mathrm{~h})$, except in those cases that where the antibiogram showed resistance. Duration of the orally administrated antibiotic treatment was not standardized, and each case was managed on an individual basis.

Brandt et al. [22] analysed 33 patients treated intravenously with beta-lactam antibiotics over 4 to 6 weeks and found a $70 \%$ accumulated probability of failure two years after the debridement was performed.

Our study seems to support the use of rifampicin for staphylococcal infections as well as the idea previously presented by Berdal et al. [26], in which the series was also treated with 3 to 7 days of intravenous antibiotics followed by 3 months of oral antibiotics that included rifampicin.

The rationale for this protocol is that in acute foreignbody related infections, two types of bacteria populations are found: planktonic bacteria that are free-floating in the joint fluid and sessile bacteria, which are those that adhere to the implant in the lower strata of the biofilm. The planktonic bacteria could be eradicated by surgical debridement and irrigation along with 7 to 10 days of antibiotic treatment. However, the sessile bacteria require antibiotics with antibiofilm activity such as rifampicin [27].

A large percentage of acute prosthetic infections are polymicrobial and, in some cases, the high reinfection rate could be caused by the presence of unidentified copathogens that are, as a result, not properly treated.
In our series, 5 cases of methicillin-resistant Staphylococcus aureus (MRSA) infections were isolated. In all of these cases the patients are in remission ( 3 cases were treated with rifampicin and linezolid and 2 cases were treated with rifampicin and clindamycin).

It has been observed that in the case of acute hematogenous infections, isolation of MRSA reduces the success rate. Among the independent factors associated with a poor prognosis in Staphylococcus aureus infections we have:

1. The need to perform a second debridement.

2. The presence of bacteraemia.

\section{3. $\quad \mathrm{CRP}>22 \mathrm{mg} / \mathrm{dl}$.}

When considering acute prosthetic knee infections due to gram-negative microorganisms, there is not much experience in terms of results with surgical debridement, implant retention and antibiotic treatment. Traditionally they have been associated with worse results. Furthermore, these microorganisms have recently been found to have increased their resistances to antibiotics.

The gram-negative bacteria that are most frequently isolated in these types of infections belong to the Enterobacteriaceae (Escherichia coli) and Pseudomona aeruginosa families. Often, gram-negative polymicrobial infections are found (58\%). After analysing the gramnegative acute prosthetic knee infections treated in our department from 2000 to 2007 with surgical debridement and implant retention, we found a $74.5 \%$ success rate [28].

The factors associated with a good prognosis in our series were:

1. A concentration $<=15 \mathrm{mg} / \mathrm{dl}$ at the time of diagnosis.

2. The use of quinolones when all of the isolated strains are sensitive to ciprofloxacine.

It is important to point out that the acute knee prosthetic joint infections due to extended-spectrum beta-lactamase (ESBL)-producing Enterobacteriaceae are associated with a worse prognosis at a 50\% [29] success rate.

Acute prosthetic knee infections caused by fungi are in the large majority of cases caused by Candida spp. These have a poor prognosis and, as a result, the complete removal of the implant is recommended in order to control the infection [6].

\section{CONCLUSION}

As we have seen, the results that can be found in the literature on treatment of acute prosthetic knee infections with surgical debridement and implant retention in association with antibiotic treatment are quite variable.

Taking into account the risk factors already mentioned, we find that the ideal candidate would be or would present:

1. A relatively young patient.

2. ASA I, II.

3. A well-fixed, stable prosthesis with no loosening.

4. Healthy soft-tissues with absence of fistulae (chronicity). 
5. Without any evidence of pus in the joint.

6. Sensitive microorganisms would be isolated (in particular to quinolones and rifampicin for grampositives and ciprofloxacin for gram-negatives).

7. No symptoms of bacteraemia.

8. CRP $<$ de $22 \mathrm{mg} / \mathrm{dl}$ in the case of gram-positive bacteria and $<15 \mathrm{mg} / \mathrm{dl}$ in the case of gram-negative bacteria.

These are the circumstances in which the highest success rate would be obtained in the case of an acute prosthetic infection treated with surgical debridement with implant retention and antibiotic treatment.

\section{CONFLICT OF INTEREST}

The authors confirm that this article content has no conflict of interest.

\section{ACKNOWLEDGEMENTS}

Declared none.

\section{REFERENCES}

[1] Backe H, Wolff D, Windsor R. Total knee replacement infection alter 2-stage reimplantation. Clin Orthop 1996; (331): 125-31.

[2] Soriano A, Bori G, García-Ramiro S, et al. Timing of antibiotic prophylaxis for primary total knee arthroplasty performed during ischemia. Clin Infect Dis 2008; 46(7): 1009-14.

[3] Wilson M, Kelley K, Thornhill T. Infection as a complication of total knee replacement arthroplasty. J Bone Joint Surg Am 1990; 72(6): $878-83$

[4] Font-Vizcarra L, Lozano L, Ríos J, Forga MT, Soriano A. Preoperative nutritional status and post-operative infection in total knee replacements: a prospective study of 213 patients. Int J Artif Organs 2011; 34(9): 876-81.

[5] Hassid VJ. Has the time come for plastic surgeons to move from povidone-iodine to chlorhexidine? Plast Reconstr Surg 2010; 126(6): 327e-8.

[6] García-Oltra E, García-Ramiro S, Martínez JC, et al. Prosthetic joint infection by Candida spp. Rev Esp Quimioter 2011; 24(1): 3741.

[7] Trampuz A, Osmon DR, Hanssen AD, Steckelberg JM, Patel R. Molecular and antibiofilm approaches to prosthetic joint infection. Clin Orthop Relat Res 2003; (414): 69-88.

[8] Tsukuyama DT, Estrada R, Gustilo RB. Infection after total hip arthroplasty. A study of the treatment of one hundred and six infections. J Bone Joint Surg Am 1996; 78-A(4): 512-23.

[9] Koyonos L, Zmistowski B, Della Valle CJ, Parvizi J. Infection control rate of irrigation and débridement for periprosthetic joint infection. Clin Orthop Relat Res 2011; 469(11): 3043-8.

[10] Greidanus NV, Masri BA, Garbuz DS, et al. Use of erythrocyte sedimentation rate and $\mathrm{C}$-reactive protein level to diagnose infection before revision total knee arthroplasty. A prospective evaluation. J Bone Joint Surg Am 2007; 89(7): 1409-16.

[11] Sastre S, Soriano A, Garcia S, Martínez J, Suso S, Mensa J. Serum C-reactive protein as predictor of infected arthroplasty. Eur J Orthop Surg Traumatol 2006; 16(1): 17-9.

[12] Jacovides CL, Parvizi J, Adeli B, Jung KA. Molecular markers for diagnosis of periprosthetic joint infection. J Arthroplasty 2011; 26(6 Suppl): 99-103.
[13] Cuñé J, Soriano A, Martínez JC, García S, Mensa J. A superficial swab culture is useful for microbiologic diagnosis in acute prosthetic joint infections. Clin Orthop Relat Res 2009; 467(2): 531-5.

[14] Ghanem E, Parvizi J, Burnett RS, et al. Cell count and differential of aspirated fluid in the diagnosis of infection at the site of total knee arthroplasty. J Bone Joint Surg Am 2008; 90(8): 1637-43.

[15] Parvizi J. New definition for periprosthetic joint infection. Am J Orthop 2011; 40(12): 614-5.

[16] Mahmud T, Lyons MC, Naudie DD, Macdonald SJ, McCalden RW. Assessing the gold Standard: A review of 253 two-Stage revisions for infected TKA. Clin Orthop Relat Res 2012; 470(10): 2730-6.

[17] Ghanem E, Parvizi J, Clohisy J, Burnett S, Sharkey PF, Barrack R. Perioperative antibiotics should not be withheld in proven cases of periprosthetic infection. Clin Orthop Relat Res 2007; 461: 44-7.

[18] Muñoz-Mahamud E, García S, Bori G, et al. Comparison of a lowpressure and a high-pressure pulsatile lavage during débridement for orthopaedic implant infection. Arch Orthop Trauma Surg 2011; 131(9): 1233-8.

[19] Bradbury T, Fehring TK, Taunton M, et al. The fate of acute methicillin-resistant Staphylococcus aureus periprosthetic knee infections treated by open debridement and retention of components. J Arthroplasty 2009; 24(6 Suppl): 101-4

[20] Segawa H, Tsukayama DT, Kyle RF, Becker DA, Gustilo RB. Infection after total knee arthroplasty. A retrospective study of the treatment of eighty-one infections. J Bone Joint Surg Am 1999; 81(10): 1434-45

[21] Mont MA, Waldman B, Banerjee C, Pacheco IH, Hungerford DS Multiple irrigation, debridement, and retention of components in infected total knee arthroplasty. J Arthroplasty 1997; 12(4): 426-33.

[22] Brandt CM, Sistrunk WW, Duffy MC, et al. Staphylococcus aureus prosthetic joint infection treated with debridement and prosthesis retention. Clin Infect Dis 1997; 24(5): 914-9.

[23] Azzam KA, Seeley M, Ghanem E, Austin MS, Purtill JJ, Parvizi J. Irrigation and debridement in the management of prosthetic joint infection: traditional indications revisited. J Arthroplasty 2010; 25(7): 1022-7.

[24] Vilchez F, Martínez-Pastor JC, García-Ramiro S, et al. Outcome and predictors of treatment failure in early post-surgical prosthetic joint infections due to Staphylococcus aureus treated with debridement. Clin Microbiol Infect 2011; 17(3): 439-44.

[25] Deirmengian C, Greenbaum J, Stern J, et al. Open debridement of acute gram-positive infections after total knee arthroplasty. Clin Orthop Relat Res 2003; 416: 129-34.

[26] Berdal JE, Skråmm I, Mowinckel P, Gulbrandsen P, Bjørnholt JV. Use of rifampicin and ciprofloxacin combination therapy after surgical debridement in the treatment of early manifestation prosthetic joint infections. Clin Microbiol Infect 2005; 11(10): 8435

[27] Zimmerli W, Frei R, Widmer AF, Rajacic Z. Microbiological tests to predict treatment outcome in experimental device-related infections due to Staphylococcus aureus. J Antimicrob Chemother 1994; 33(5): 959-67.

[28] Martínez-Pastor JC, Muñoz-Mahamud E, Vilchez F, et al. Outcome of acute prosthetic joint infections due to gram-negative bacilli treated with open debridement and retention of the prosthesis. Antimicrob Agents Chemother 2009; 53(11): 4772-7.

[29] Martínez-Pastor JC, Vilchez F, Pitart C, Sierra JM, Soriano A Antibiotic resistance in orthopaedic surgery: acute knee prosthetic joint infections due to extended-spectrum beta-lactamase (ESBL)producing Enterobacteriaceae. Eur J Clin Microbiol Infect Dis 2010; 29(8): 1039-41. 